\title{
Trombo-embolismo pulmonar: necesitamos sospecha clínica
}

\section{Pulmonary thromboembolism: clinical suspicion required}

\author{
M.T. García-Sanz ${ }^{1}$, C. Pena-Álvarez ${ }^{2}$, F.J. González-Barcala ${ }^{3}$
}

\section{RESUMEN}

La enfermedad tromboembólica venosa (ETV) es un importante problema de salud, con una incidencia anual de 1-2 casos /1.000 personas. Un tercio de los pacientes con ETV sintomática cursan con tromboembolismo pulmonar (TEP), que es frecuentemente infradiagnosticado debido a su presentación clínica variable e inespecífica. El conocimiento de los signos y síntomas de la ETV puede reducir los retrasos en el diagnóstico y, con ello, la morbimortalidad asociada. Sin embargo, el TEP se puede presentar con un amplio espectro clínico, desde apenas síntomas hasta shock o inestabilidad hemodinámica, por lo que no debemos limitar la sospecha de TEP a aquellos pacientes con presentación típica; además esta posibilidad diagnóstica debe ser considerada no sólo ante la presencia de síntomas agudos, sino también en aquellos pacientes con síntomas respiratorios de evolución prolongada.

Palabras clave. Tromboembolismo pulmonar. Diagnóstico diferencial. Síndromes clínicos. Síntomas frecuentes. Retraso diagnóstico.

\begin{abstract}
Venous thromboembolism (VTE) is a major health problem, with an annual incidence of 1-2 per 1,000 person-years. One in three patients with symptomatic VTE presents pulmonary embolism (PE), which is often underdiagnosed due to variable and nonspecific clinical presentation. Knowing the signs and symptoms of VTE can reduce delays in diagnosis, and thus VTErelated morbidity and mortality as well. Nevertheless, $\mathrm{PE}$ can present a wide clinical spectrum, ranging from being asymptomatic to shock or hemodynamic instability, which is why PE suspicion should not be limited to those patients with typical presentation. Therefore, this diagnostic possibility should not only be considered for patients with acute symptoms, but also for those with persistent respiratory symptoms.
\end{abstract}

Key words. Pulmonary embolism. Differential diagnosis. Clinical syndromes. Common symptoms. Delayed diagnosis.
1. Servicio de Urgencias. Hospital do Salnés. Vilagarcía de Arousa.

2. Servicio de Oncología. Complejo Hospitalario de Pontevedra.

3. Servicio de Neumología. Complejo Hospitalario Clínico Universitario. Santiago de Compostela.

Recepción: 29 de noviembre de 2011

Aceptación provisional: 12 de enero de 2012

Aceptación definitiva: 19 de enero de 2012

\section{Correspondencia:}

MT García Sanz

Servicio de Urgencias.

Hospital do Salnés

Ande-Rubiáns s/n

36600 Vilagarcía de Arousa. Pontevedra. España

E-mail: teresa.garcia.sanz@sergas.es 


\section{TROMBOEMBOLISMO PULMONAR}

La enfermedad tromboembólica venosa (ETV) es un importante problema de salud, con una incidencia anual de 1-2 casos $/ 1.000$ personas que aumenta en un $1 \%$ por año de edad en los mayores de 75 años ${ }^{1,2}$. Un tercio de los pacientes con ETV sintomática cursan con tromboembolismo pulmonar (TEP) ${ }^{3}$, que además es la manifestación más grave ${ }^{4}$ y tiene una mortalidad del $30 \%{ }^{5}$. Además, el TEP es frecuentemente infradiagnosticado (sólo la tercera parte de los que se producen ${ }^{5}$ ) debido a que su presentación clínica es variable e inespecífica. La asociación de incidencia elevada e infradiagnóstico frecuente convierte a esta enfermedad en un importante reto diagnóstico. Por ello, para identificar a los pacientes en los cuales es necesaria la realización de pruebas diagnósticas, es fundamental un alto nivel de sospecha clínica.

El conocimiento de los signos y síntomas de la ETV puede reducir los retrasos en el diagnóstico ${ }^{6} \mathrm{y}$, con ello, la morbimortalidad asociada ${ }^{7}$. En un $90 \%$ de los casos la sospecha de TEP se plantea en base a signos $\operatorname{clínicos}^{8}$, junto a la presencia de factores de riesgo, presentes en el 75\%-94\% de los $\operatorname{casos}^{9,10}$.

El TEP se puede presentar con un amplio espectro clínico, desde apenas síntomas hasta shock o inestabilidad hemodinámica, variables en función de la gravedad ${ }^{11}$. Los síntomas más habituales son disnea, que puede ser de reposo o de esfuerzo, y dolor torácico de características pleuríticas. Otros síntomas son tos, generalmente irritativa, aunque se han descrito casos con esputo mucoso y purulento, dolor o edema en extremidades inferiores, hemoptisis, dolor torácico anginoso, ortopnea y palpitaciones $^{9-11}$. Un porcentaje variable de pacientes tiene fiebre ${ }^{11,12}$. Síncope y presíncope son frecuentes en TEP con repercusión hemodinámica ${ }^{13}$ (Tabla 1).

Tabla 1. Síntomas y signos en pacientes con sospecha de $\mathrm{TEP}^{9,11,18,31,33}$

\begin{tabular}{|l|c|l|c|}
\hline \multicolumn{1}{|c|}{ Síntomas } & $\%$ & \multicolumn{1}{c|}{ Signos Clínicos } & \% \\
\hline Disnea & $79-80$ & Taquipnea & $26-64$ \\
\hline Disnea de reposo & $50-61$ & Taquicardia & $7-11$ \\
\hline Disnea de esfuerzo & $16-27$ & Diaforesis & $2-10$ \\
\hline Ortopnea & 36 & Fiebre & 15 \\
\hline Dolor pleurítico & $39-52$ & Incremento del 2o tono cardíaco & 13 \\
\hline Dolor torácico no pleurítico & $15-38$ & Ingurgitación yugular & $1-11$ \\
\hline Tos & $20-43$ & Cianosis & $8-21$ \\
\hline Hemoptisis & $8-15$ & Crepitantes & $3-31$ \\
\hline Síncope & $5-19$ & Sibilancias & 5 \\
\hline Mareo & 12 & Roncus & 21 \\
\hline Dolor o edema en pantorrilla & 39 & Disminución del murmullo vesicular & $15-47$ \\
\hline Dolor o edema en muslo & $6-42$ & Signos de TVP & \\
\hline
\end{tabular}

En la exploración física, taquipnea y taquicardia son los hallazgos más frecuentes. Puede encontrarse, además, refuerzo en $4^{\circ}$ y $2^{\circ}$ tonos, ingurgitación yugular y galope derecho (frecuente en TEP con repercusión hemodinámica) ${ }^{13}$. La ausculta- ción pulmonar suele ser normal, siendo los crepitantes y la disminución del murmullo vesicular las alteraciones más frecuentes ${ }^{9}$. En el 17\% de los casos la palpación torácica puede ser dolorosa ${ }^{14}$. El $32 \%$ de los pacientes con TEP refieren dolor a la palpa- 
ción de las pantorrillas ${ }^{9}$, y en el $40-50 \%$ de los pacientes con TEP sintomático puede demostrarse TVP mediante eco-doppler, que afecta a las venas proximales en $2 / 3$ de los $\operatorname{casos}^{12}$ (Tabla 1). Si un paciente no presenta disnea, taquipnea o dolor torácico pleurítico el TEP es poco probable; sin embargo, el 3-7\% de los pacientes con TEP están completamente asintomáticos en el momento inicial ${ }^{12}$.

La combinación de síntomas y signos permite establecer la sospecha de TEP. Tradicionalmente, los síntomas iniciales del TEP agudo en pacientes sin enfermedad cardiopulmonar subyacente han sido agrupados en 3 síndromes en base a las características clínicas ${ }^{9,15-17}$ :

1. Síndrome de colapso circulatorio, definido como síncope o tensión arterial sistólica (TAS) $\leq 90 \mathrm{~mm} \mathrm{Hg}^{18}$. Se produce como consecuencia de una sobrecarga aguda suficiente para superar la capacidad adaptativa del ventrículo derecho. En estos pacientes es frecuente encontrar taquicardia, hipoxemia, alteraciones electrocardiográficas, cardiomegalia ${ }^{19} \mathrm{e}$ ingurgitación yugular ${ }^{20}$.

2. Síndrome hemoptisis/dolor pleurítico (antes llamado infarto pulmonar). Presente en los que cursan con hemoptisis o dolor pleurítico en ausencia de colapso circulatorio; es la presentación más frecuente ${ }^{9}$. En este contexto clínico es más frecuente la presencia de derrame pleural, atelectasias, infiltrados pulmonares en la radiografía de tórax ${ }^{19} \mathrm{y}$ auscultación de roce pleural ${ }^{21}$.

3. Síndrome de disnea no complicada. Consiste en disnea en ausencia de hemoptisis, dolor pleurítico o colapso circulatorio. En su presentación clínica se ve mayor frecuencia de taquicardia, hipoxemia, anormalidades electrocardiográficas y cardiomegalia o redistribución vascular en la radiografía de tórax ${ }^{19}$. Los pacientes suelen ser de mayor edad, tienen más frecuentemente neoplasia subyacente y el factor desencadenante es habitualmente la inmovilización por causas médicas ${ }^{20}$.

La estratificación por síndromes no sólo puede ser de ayuda en el abordaje diagnóstico de la enfermedad, sino que incluso tiene cierto valor pronóstico, ya que permite identificar a aquellos pacientes con mayor riesgo de muerte, en los que las dosis y la duración del tratamiento deberían ser diferentes ${ }^{19}$. Así, los pacientes con TEP que cursan con colapso circulatorio o con disnea aislada tienen mayor riesgo de muerte y, además, parece que la presentación clínica inicial también se asocia con la gravedad clínica de la recurrencia del TEP ${ }^{19}$. Algunos estudios refieren que, aunque la frecuencia de recidivas no es diferente, la mortalidad de éstas es mayor en los que habían presentado colapso circulatorio ${ }^{20}$. Tras el primer episodio, el $60 \%$ de los casos recurren y las tasas de mortalidad en esos pacientes son especialmente elevadas (4-9\%). Tanto en el primer episodio como en la recurrencia los síntomas más frecuentes suelen ser disnea o dolor torácico ${ }^{8}$. Los que sobreviven a la embolia pulmonar sufren de hipertensión pulmonar y ocasionalmente fallecen como consecuencia de insuficiencia cardíaca derecha años después ${ }^{22}$.

En los últimos años se tiende a sustituir la clasificación en síndromes por otra en función de la situación hemodinámica, que diferencia entre TEP masivo, submasivo y no masivo. El TEP masivo se caracteriza por inestabilidad hemodinámica. El submasivo se define por la presencia de disfunción ventricular derecha, pero sin inestabilidad hemodinámica. El resto se consideran no masivos ${ }^{23,24}$.

La posibilidad de TEP masivo debe ser tenida en cuenta en los pacientes con síncope, hipotensión, hipoxemia extrema, disociación electromecánica o parada cardia$\mathrm{ca}^{21}$. El tamaño del émbolo y el calibre de la arteria pulmonar ocluida tienen influencia significativa en el impacto hemodinámico y en el compromiso cardiopulmonar del TEP agudo. El aumento de presión en el ventrículo derecho conduce a dilatación, disfunción e isquemia del mismo, y el fallo cardíaco derecho predispone a inestabilidad hemodinámica y shock cardiogénico ${ }^{18}$. 
Los casos con mayor repercusión hemodinámica se diagnostican antes ${ }^{25}$, pero tienen un peor pronóstico a corto plazo, siendo la presencia de hipotensión el mejor predictor de evolución desfavorable ${ }^{26}$. La presencia de taquicardia, ingurgitación yugular, refuerzo del cierre de la pulmonar y hepatomegalia sugieren disfunción ventricular, y son datos que podrían ayudar a identificar a aquellos pacientes con TEP submasi$\mathrm{vo}^{27}$. Los pacientes con TEP con cifras de TA normales y sin disfunción de ventrículo derecho tienen excelente pronóstico únicamente con terapia anticoagulante ${ }^{27}$. Sin embargo, los síntomas pueden ser leves o estar ausentes en pacientes con embolismos extensos en arterias segmentarias ${ }^{9}$, los trombos periféricos de gran tamaño pueden cursar silentes y émbolos de menor calibre pueden asociarse con síntomas graves, sobre todo en pacientes con enfermedades cardíacas previas ${ }^{21}$.

La probabilidad clínica de TEP debe ser establecida de manera individualizada para cada paciente. La utilización de escalas de probabilidad clínica validadas permite homogenizar el enfoque diagnóstico entre médicos con diferentes niveles de experiencia clínica ${ }^{28,29}$. Pero, al realizar comparaciones entre las escalas más utilizadas (Wells y Ginebra) se evidencia la dificultad del diagnóstico de esta enfermedad, ya que clasificarían de manera diferente al $45 \%$ de pacientes $^{30}$.

De los pacientes que ingresan tras consultar por disnea aguda, dolor torácico, síncope o palpitaciones, sólo en el $4 \%$ de los casos es debido a TEP; mientras que el resto presentarán otras enfermedades como insuficiencia cardíaca, neumonía o agudización de enfermedad pulmonar obstructiva crónica (EPOC). Consideramos que la evidencia de estas dificultades refuerza la necesidad de establecer una sospecha clínica, pues los avances en los recursos tecnológicos son poco útiles si no se considera la posibilidad diagnóstica de TEP, como han mostrado distintos estudios sobre autopsias ${ }^{31}$.

El retraso en el diagnóstico del TEP, definido como el tiempo transcurrido entre la aparición del primer síntoma y el diag- nóstico de la enfermedad, es resultado de la suma del tiempo que el paciente tarda en recibir valoración clínica tras el inicio de los síntomas y del tiempo entre esta primera atención médica y la confirmación diagnóstica.

Diversos estudios han investigado factores clínicos y demográficos y su asociación con retrasos en la consulta:

1. Los pacientes pueden retrasar la solicitud de evaluación médica debido a que el TEP cursa con clínica similar a la que presentan durante la agudización de comorbilidades ${ }^{32}$ como la enfermedad cardíaca, en especial en aquellos con disnea de instauración progresiva $^{25}$.

2. Los fumadores activos también tardan en consultar, probablemente por considerar que sus síntomas y enfermedades pulmonares son debidos al tabaco $^{32}$.

3. La valoración de los síntomas influye en el retraso, por ejemplo si el paciente considera que no son lo suficientemente serios como para acudir al hospital ${ }^{33}$.

4. Un nivel educativo elevado también se asocia con mayor retraso en la consulta $^{32,33}$.

En cambio, son motivos de consulta precoz la importancia otorgada a un síntoma concreto, como el síncope, la disnea súbita $^{25}$, la hipotensión o la frecuencia respiratoria elevada ${ }^{33}$; así como la percepción de gravedad por parte del paciente ${ }^{34}$. Los casos con antecedente de TEP probablemente consultan antes porque recuerdan los síntomas del episodio anterior ${ }^{25}$.

Otro punto de especial interés es el retraso en la confirmación diagnóstica, que puede ser debido a diversos motivos:

1. La inespecificidad de los síntomas del TEP, bajas puntuaciones en escala de Wells o ausencia de síncope o disnea súbita dan lugar a diagnósticos erróneos ${ }^{25}$.

2. La presencia de comorbilidades puede enmascarar los síntomas de TEP ${ }^{35}$, planteándose diagnósticos alterna- 
tivos en pacientes con disnea como la insuficiencia cardíaca o la agudización de la EPOC y, en los pacientes con palpitaciones, la fibrilación auricular ${ }^{31}$.

3. El haber recibido previamente atención sanitaria aumenta el retraso diagnóstico ${ }^{32}$.

4. El uso de anticonceptivos orales ${ }^{35}$.

Sin embargo, no hay suficiente evidencia para afirmar que la edad y la presentación atípica del TEP en los pacientes ancianos se relacionen con retrasos en el diagnóstico ${ }^{36}$. Influyen en la precocidad del diagnóstico: la presencia de determinados síntomas, como síncope, hipotensión ${ }^{32}$ o disnea de aparición súbita ${ }^{25}$; la existencia de patologías previas, como cáncer e ictus $^{35}$; la presencia de factores de riesgo transitorios (cirugía o traumatismo recientes, inmovilización, embarazo) $)^{32,34}$; o la disponibilidad de pruebas complementarias, como la TC helicoidal ${ }^{33}$.

En resumen, los médicos no deben limitar la sospecha diagnóstica de TEP a aquellos pacientes con presentación típica, y esta posibilidad diagnóstica debe ser considerada no sólo ante la presencia de síntomas agudos, sino también en aquellos pacientes con síntomas respiratorios de evolución prolongada.

\section{BIBLIOGRAFÍA}

1. OGer E. Incidence of venous thromboembolism: a community-based study in Western France. EPI-GETBP Study Group. Groupe d'Etude de la Thrombose de Bretagne Occidentale. Thromb Haemost 2000; 83: 657-660.

2. Silverstein M, Heit J, Mohr D, Petterson TM, O'FALlon WM, MeLton III LJ. Trends in the incidence of deep vein thrombosis and pulmonary embolism: a 25-year population-based study. Arch Intern Med 1998; 158: 585-593.

3. White R. The epidemiology of venous thromboembolism. Circulation 2003; 107: 14-18.

4. Monreal M, Ruiz J, Fraile M, Bonet M, Davant E, Machart J et al. Prospective study on the usefullness of lung scan in patients with deep vein thrombosis of de lower limbs. Thromb Haemost 2001; 85: 771-774.
5. Iribarren-Sarrías J, Martínez-Riera A. Tromboembolismo pulmonar. Medicine 2001; 8: 25872592.

6. Elliot C, Goldhaber S, Jensen R. Delays in diagnosis of deep vein thrombosis and pulmonary embolism. Chest 2005; 128: 3372-3376.

7. Dalen J. Pulmonary embolism: what have we learned since Virchow? Chest 2002; 122: 1440-1446.

8. Berghaus T, VonScheidt W, Schwaiblmair M. Time between first symptoms and diagnosis in patients with acute pulmonary embolism: era patients with recurrent episodes diagnosed earlier? Clin Res Cardiol 2011; 100: 117-119.

9. Stein P, Beemath A, Matta F, Weg J, Yusen R, HaLES $\mathrm{C}$ et al. Clinical characteristic of patients with acute pulmonary embolism: data from PIOPED II. Am J Med 2007; 120: 871-879.

10. Heit J, O'Fallon W, Petterson T, Lohse C, SilversTEIN M, MoHR D et al. Relative impact of risk factors for deep vein thrombosis and pulmonary embolism: a population-based study. Arch Intern Med 2002; 162: 1245-1248.

11. Pollack C, Schreiber D, Goldhaber S, Slaterry D, FAnIKos J, O'NeIL B et al. Clinical characteristics, management, and outcomes of patients diagnosed with acute pulmonary embolism in the emergency department. J Am Coll Cardiol 2011; 57: 700-706.

12. Agarwal R, Varma S. Acute pulmonary embolism. Eastern Journal of Medicina 2009; 14: 57-68.

13. Uresandi F, Blanquer J, Conget F, Gregorio Md, Lobo J, Otero R et al. Guía para el diagnóstico, tratamiento y seguimiento de la tromboembolia pulmonar. Arch Bronconeumol 2004; 40: 580-594.

14. LeGal G, Testuz A, Righini M, Bounameaux H, PeRRIER A. Reproduction of chest pain by palpation: diagnostic accuracy in suspected pulmonary embolism. BMJ 2005; 330: 452-453.

15. Stein P, Willis P, deMets D. History and physical examination in acute pulmonary embolism in patients without preexisting cardiac or pulmonary disease. Am J Cardiol 1981; 47: 218-223.

16. Stein P, Terrin M, Hales C, Palevsky Hi, Saltzman HA, Thоmpson вт et al. Clinical, laboratory, roentgenographic, and electrocardiographic findings in patients with acute pulmonary embolism and no pre-existing cardiac or pulmonary disease. Chest 1991; 100: 598-603.

17. Stein P, Henry J. Clinical characteristics of patients with acute pulmonary embolism stratified according to their presenting syndromes. Chest 1997; 112: 974-979. 
18. Rendina D, Bonis SD, Gallotta G, Piedimonte V, Mossetti G, FiLIPPo GD et al. Clinical, historical and diagnostic findings asociated with right ventricular dysfuntion in patients with central and non-massive pulmonary embolism. Intern Emerg Med 2010; 5: 53-59.

19. Lobo J, Zorrilla V, Aizpuru F, Uresandi F, GarcíaBragado F, Conget $\mathrm{F}$ et al. Clinical syndromes and clinical outcome in patients with pulmonary embolism. Findings from the RIETE Registry. Chest 2006; 130: 1817-1822.

20. Lobo-Beristain J, Santaolalla CE. Síndromes clínicos de embolia pulmonar. Semin Fund Esp Reumatol 2006; 7: 139-146.

21. TAPSON V. Acute pulmonary embolism. N Engl J Med 2008; 358: 1037-1052.

22. Pengo V, Lensing A, Prins M, Marchiori A, DavidSON BL, Tiozzo F et al. Incidence of chronic thromboembolic pulmonary hypertension after pulmonary embolism. N Engl J Med 2004; 250: 2257-2264.

23. Kucher N, Luder C, Dornhofer T, Windecker S, Meier B, Hess O. Novel management strategy for patients with suspected pulmonary embolism. Eur Heart J 2003; 24: 366-376.

24. Torbicki A, Perrier A, Konstantinides S, Agnelli G, Nazzareno G, Pruszczyk P et al. Guidelines on the diagnosis and management of acute pulmonary embolism: the Task Force for the Diagnosis and Management of Acute Pulmonary Embolism of the European Society of Cardiology (ESC). Eur Heart J 2008; 29: 22762315.

25. Alonso-Martínez J, Sánchez F, Echezarreta M. Delay and misdiagnosis in sub-massive and non-massive acute pulmonary embolism. Eur J Intern Med 2010; 21: 278-282.

26. Marshall P, Matthews K, Siegel M. Diagnosis and management of life-threatening pulmonary embolism. J Intensive Care Med 2011; 26: 275-294.

27. Piazza G, Goldhaber S. Management of submasive pulmonary embolism. Circulation 2010; 122: 1124-1129.
28. Blondon M, Gal GL, Righini M. Stratégie diagnostique et intérêt comparatif des scores cliniques pour le diagnostic démbolie pulmonaire. Rev Med Interne 2010; 31: 742-749.

29. Ceriani E, Combescure C, Le Gal G, Nendaz M, Perneger T, Bounameaux $\mathrm{H}$ et al. Clinical prediction rules for pulmonary embolism: a systematic review and meta-analysis. J Thromb Haemost 2010; 8: 957-970.

30. KLoK F, Kruisman E, SpaAn J, NiJkeuter M, Righini M, AuJEsky D et al. Comparison of the revised Geneva score with the Wells rule for assessing clinical probability of pulmonary embolism. J Thromb Haemost 2008; 6: 40-44.

31. Squizzato A, Luciani D, Rubboli A, Gennaro L, LANDOLFI R, LuCA CD et al. Differential diagnosis of pulmonary embolism in outpatients with non-specific cardiopulmonary symptoms. Intern Emerg Med 2011 Nov 18. [Epub ahead of print].

32. Bulbul Y, AyiK S, Oztuna F, Ozlu T, Sahin S. The relationship between socio-demographic characteristics of patients and diagnostic delay in acute pulmonary thromboembolism. Ups J Med Sci 2011; 116: 72-76.

33. Bulbul Y, Oszu S, Kosucu P, Oztuna F, Ozlu T. Time delay between onset of symptoms and diagnosis in pulmonary embolism. Respiration 2009; 78: 36-41.

34. Ageno W, Agnelli G, Imberti D, Moia M, PalareTI G, PISTELli R et al. Factors associated with the timing of diagnosis of venous thromboembolism: results from the MASTER registry. Thromb Res 2008; 121: 751-756.

35. Ozsu S, Oztuna F, Bulbul Y, Topbas M, Ozlu T, Kosucu $\mathrm{P}$ et al. The role of risk factors in delayed diagnosis of pulmonary embolism. Am J Emerg Med 2011; 29: 26-32.

36. Bergaus T, Thilo C, Schidt Wv, Schwaiblmair M. The impact of age on the delay in diagnosis in patients with acute pulmonary embolism. Clinical and applied Thrombosis/Hemostasis 2011. DOI: 10.1177/1076029611404218. 